\title{
Case report: left upper and left lower sleeve lobectomies with robotic surgery
}

\author{
Alper Toker ${ }^{1}$, Erkan Kaba ${ }^{2}$, Robert Herron ${ }^{1}$, Kemal Ayalp ${ }^{2}$ \\ ${ }^{1}$ Department of Cardiovascular and Thoracic Surgery, Heart and Vascular Surgery Institute, West Virginia University Hospital, Morgantown, USA; \\ ${ }^{2}$ Group Florence Nightingale Hospitals, Department of Thoracic Surgery, Istanbul Florence Nightingale Hospital, Istanbul, Turkey \\ Correspondence to: Alper Toker, MD. 1 Medical Center Drive Box 8500, Morgantown, WV 26506, USA. \\ Email: salpertoker@gmail.com; alper.toker@wvumedicine.org.
}

Background: Surgical resection provides the highest possible survival in non small cell lung cancer (NSCLC). To allow negative margins for tumors extending into the lobar bronchus, sleeve resections can be performed with the potential for better early and late outcomes. This would also include an invasive N1 lymph node. Because of better flexibility due to endowrist instruments and high definition with threedimensional viewing sleeve resections could be performed with the surgical robot.

Case Description: Two cases of left sleeve lobectomies, one left lower and one left upper, will be described in this study. Both of the bronchial anastomoses were performed with Vi-Loc 3-0 barbed suture (Covidien, Mansfield, MA, USA). We prefer to use 3-0 V lock, 180 needle size 26 and 15 mm sutures. The results of our study suggest that when sleeve resection is required during surgery for lung cancer, the procedure can be safely performed with robot-assisted thoracoscopic surgery.

Conclusions: In the classical knowledge for minimally invasive surgery, conversion to open surgery was recommended in the previous decade. Some reports with VATS have been presented for sleeve resections. However, utilization of this technique has been a rare indication. Based on our experience, we claim that conversion to thoracotomy would not be required if the surgical robot has been used. In this manuscript, literature findings were discussed.

Keywords: Sleeve; robotic surgery; left; case report

Received: 06 January 2020; Accepted: 23 July 2020; Published: 20 April 2022.

doi: 10.21037/jovs-2020-04

View this article at: http://dx.doi.org/10.21037/jovs-2020-04

\section{Introduction}

Surgical resection provides the highest possible survival in early stage and locally advanced non-small cell lung cancer (NSCLC) when combined with oncological therapy (1). In order to provide the greatest likelihood for cure, the resection should be complete (2). Complete surgical resection in a lung cancer patient requires all of the following: microscopically tumor free resection margins, systematic nodal lymph node dissection, no extracapsular nodal extension of the tumor, and the highest mediastinal node removed must be negative for malignancy (3). Whenever there is involvement of the resection margin, there is extracapsular nodal invasion, there are retained positive lymph nodes, there are malignant pleural and/or pericardial effusions, the resection is defined as incomplete (3). If the resection margins are free and no residual tumor is left behind, but there is carcinoma in situ at the bronchial margin, or positive pleural lavage cytology, the term uncertain resection is proposed (3).

The presence of microscopically identified tumor cells at the bronchial stump is associated with higher recurrence rate and worse survival rates (4). Tumours involving the proximal lobar bronchi are a matter of concern in minimally invasive resection after surgery for lung cancer. In such circumstances, we recommend not to staple the bronchus, but instead dividing the lobar bronchus without stapling and closing the bronchus with primary suture closure to provide 
a wider surgical bronchial margin. Alternative resections in such scenarios include pneumonectomy or bilobectomy. However, in order to obtain negative margins for tumors extending into the lobar bronchus, sleeve resections can be performed with the potential for better early and late outcomes (5).

The indications of a sleeve resection include: a centrally located distinct malignancy, intraluminal tumour infiltrations of peribronchial or extrabronchial areas, and for lymph nodal involvement of lymph nodes (N1) within the area of resection (6). Endobronchial carcinoid tumours or benign lesions such as endobronchial hamartomas can also serve as indications for bronchial sleeve resections $(7,8)$. Benign bronchial strictures related to trauma or inflammatory disease, airway issues following lung transplantation, acute traumatic disruption of the airway, and metastatic malignancies with lobar extension via the main bronchus also constitute indications for pulmonary sleeve resections. Approximately $8 \%$ of the resections for primary lung cancer are sleeve resections (9). It is reported that the most common and technically easiest sleeve resection is right upper sleeve lobectomy (9).In our experience with robotic surgery, most of the sleeve lobectomies are right upper lobe sleeves; although, as of now, sleeve lobectomies for all single lobes exist (10).

Preoperative bronchoscopy is paramount when identifying candidates for sleeve. In cases where bronchoscopy may be unable to identify such patients, frozen section analysis guides the surgeon in regards to the presence or absence of extrabronchial tumor involvement, as well as the presence of extracapsular lymph node invasion into the bronchus.

Advanced laparoscopic suturing methods are generally regarded as an indicator of high performance among experienced minimally invasive surgeons (11). In lung surgery, due to various type of sutures and suture techniques required for a sleeve resection, the surgical technique is viewed as being a more complex operation when compared to a standard lobectomy (12). We present the following article in accordance with the CARE reporting checklist (available at https://jovs.amegroups. com/article/view/10.21037/jovs-2020-04/rc).

\section{Case presentation}

\section{Standard preparations for a possible left robotic sleeve resection}

The contrast enhanced chest computerized tomography
(CT) is a golden tool to understand the possible need for a sleeve resection. A careful surgeon should pay attention to the tumor invading the lobar bronchus and one of the lobar arteries at the origin on the main pulmonary artery. This condition requires a double sleeve lobectomy or a patch angioplasty operation to the pulmonary artery in addition to the bronchial sleeve lobectomy. This intervention is required mainly in left upper lobe sleeve lobectomies. Before performing a sleeve lobectomy, we recommend to perform a lobectomy where the bronchus is transected with a knife. If the margin is positive, the bronchial resection could be converted to a sleeve lobectomy.

In this operation, a standard double lumen endotracheal tube is used. We prefer to place a left sided tube in all patients undergoing this operation. Bronchoscopy is required to correctly place and control the position of the tube, especially after the patient is positioned in the lateral decubitus position. The table is set according to the DaVinci SI position, the patient is placed in the right lateral decubitus position, and the table is then flexed and 4 ports are placed. Three ports at $8^{\text {th }}$ intercostal space and an access port to $5^{\text {th }}$ intercostal anterior axillary line are our preferences. The most anterior port serves as assistant port and left arm port. We generally place an Alexis soft tissue retractor (Applied Medical California, USA) here. From this port Table surgeon assists with suctioning and retractions. The technique presented here is not a completely portal robotic surgery technique. This technique could be named as Robot assisted thoracoscopic sleeve lobectomy. We then place and position the left arm of the robot to the superior angle of the Alexis port (Figure 1).

Recently, we have started to perform our cases with the Da Vinci Xi Robotic surgery device. Xi is more easily adopted. We prefer to use $\mathrm{CO}_{2}$ insufflation and 5 ports with $\mathrm{Xi}$. In this situation we do not use soft tissue retractors.

All procedures performed in this study were in accordance with the ethical standards of the institutional and/or national research committee(s) and with the Helsinki Declaration (as revised in 2013). Written informed consent were obtained from the patients for publication of this case report and accompanying images. A copy of the written consent is available for review by the editorial office of this journal.

\section{Case 1: left lower sleeve lobectomy}

The first patient is a 20-year-old male, who was diagnosed with a carcinoid tumor at the orifice of left lower lobe (Figure 2). The patient was intubated and location of endotracheal 

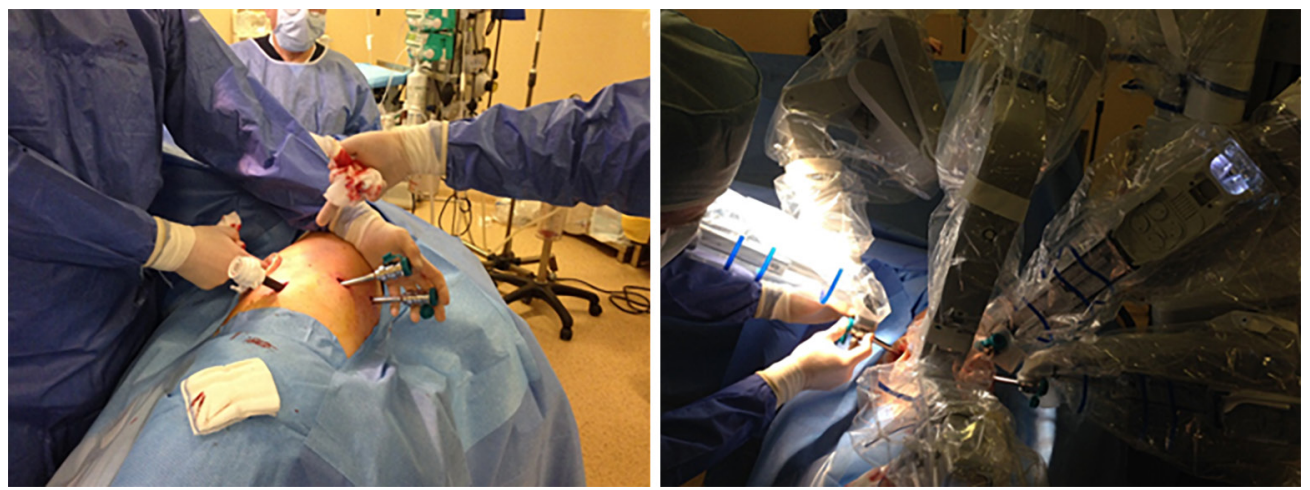

Figure 1 Ports and Docking with DaVinci SI for a left lung resection. For lower lobes, port incisions could be one intercostal below.

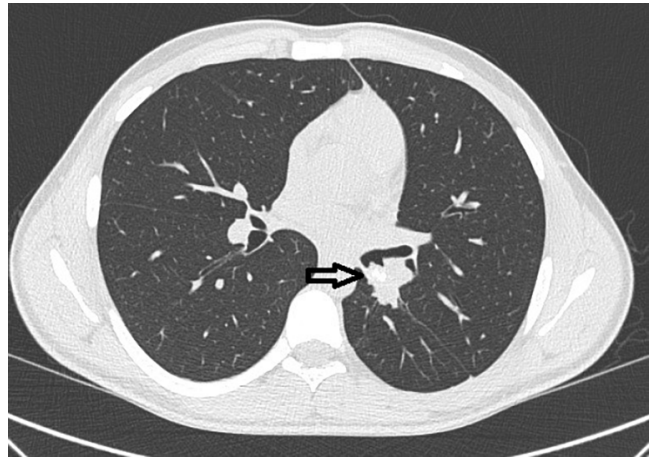

Figure 2 Left lower lobe obstruction with the carcinoid tumor is recognizable with chest computerized tomography.

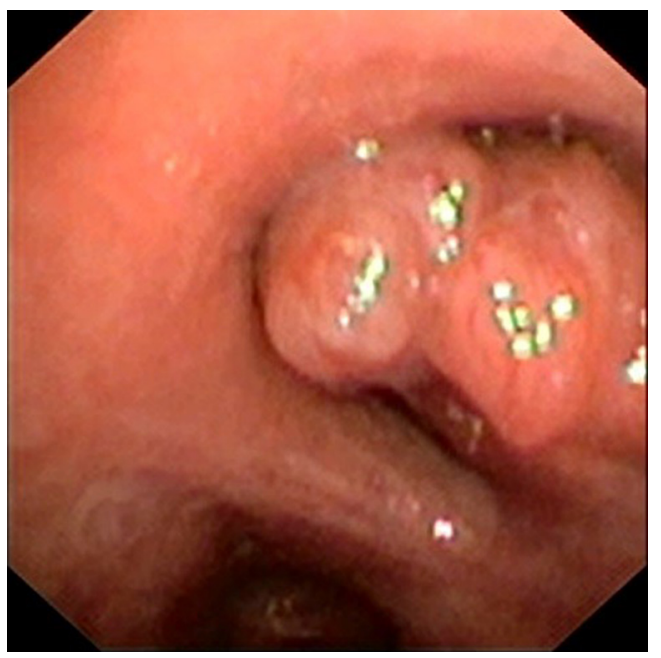

Figure 3 Bronchoscopy reveals close relationship with the resection line. tube was confirmed with fiberoptic bronchoscopy (FOB) (Figure 3). It was involving the orifice of left lower lobe and left lower lobectomy would end with positive surgical margins. The robotic ports were placed as previously described and demonstrated above. The left lower lobectomy was performed with division of artery and inferior pulmonary vein. We complete the mediastinal lymph node dissection prior to the division of the vessels in all oncologic surgeries. Stations 5, 6, and 7-11 were removed in a standard fashion along with the fatty tissue surrounding the lymph nodes.

The only remaining structure in the lobar hilum was the lower lobe bronchus. The left upper lobe bronchus was divided with a scalpel and endo-scissors. Then the left main bronchus was transected with a scalpel. A sleeve resection was performed. Frozen section pathology confirmed that both the upper and main bronchi surgical margins were negative. The anastomosis was performed with Vi-Loc 3-0 barbed suture (Covidien, Mansfield, MA, USA). We prefer to use 3-0 V lock, 180 needle size 26 and $15 \mathrm{~mm}$ sutures and generally complete the anastomosis with 2 sutures.

In all of our experiences, we place 2 sutures at the most inferior and posterior aspect of the both bronchi. We place a prostate bulldog clamp to one of them and then pull the suture. With the other suture, we perform a continuous running technique to anastomose the medial part of the bronchus. After we finish the anteromedial and a portion of the superior aspect, we continue with the posterior suture to complete the anastomosis. Both sutures are then tied to each other.

In this patient, we started with a suture and anastomosed the medial side of the bronchus and tied it with a new suture 
on the half way of the anastomosis and continued with the second suture. We approximated the size discrepancies by taking larger bites from the main bronchus and smaller bites from the upper lobe bronchus. Air leak and deflation tests were performed (Video 1). We always place a single chest tube $28 \mathrm{~F}$ after a robotic lung resection. Bronchoscopy was then performed to clean blood and secretions from the airway after the dual lumen endotracheal tube was replaced

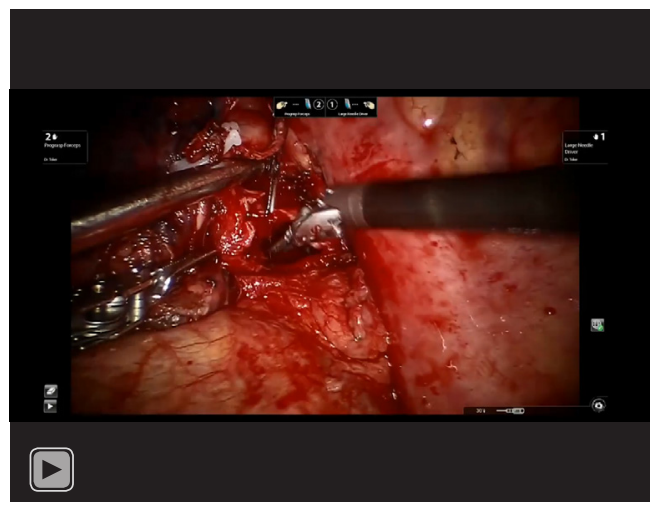

Video 1 The demonstration of the case. with a \#8 single lumen endotracheal tube before taking the patient to intensive care unit. We keep the patient one night in ICU for the extubation and close follow up in the first 12 hours after surgery. No complications occurred and patient discharged on day 7. Chest CT in the following month demonstrated a patent and satisfactory bronchial lumen (Figure 4).

\section{Case 2: left upper sleeve lobectomy}

This patient is a 61-year-old female, former smoker with a 40-pack year history, and a history of COPD. She developed hemoptysis which subsequently led to the diagnosis of a left upper lobe lung squamous cell lung cancer via FOB (Figures 5,6). On imaging, she was found to have a lymph node at station 11 that was suspected to be involved with malignancy. She also demonstrated an interstitial lung disease pattern. Her pulmonary function tests and DLCO were at the lower limits for a lung resection.

She was intubated with a double lumen, left sided endotracheal tube. The table and patient were placed as described above and ports were situated as per our standard
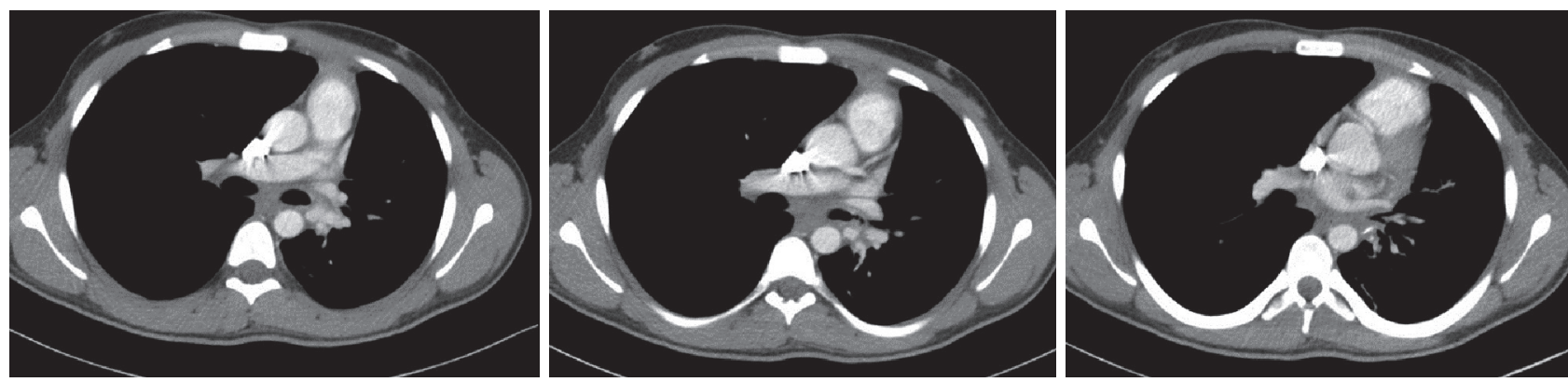

Figure 4 Post-operative chest CT of the left lower lobectomy patient.
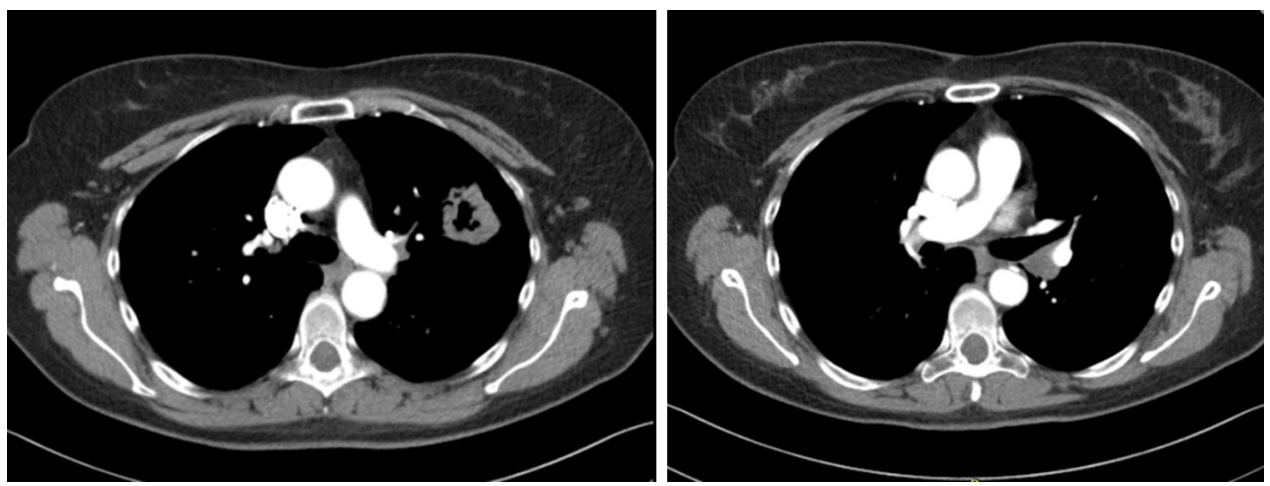

Figure 5 Left upper lobe tumor with station 11 positivity. Enlarged node found to be invasive with surgery. 


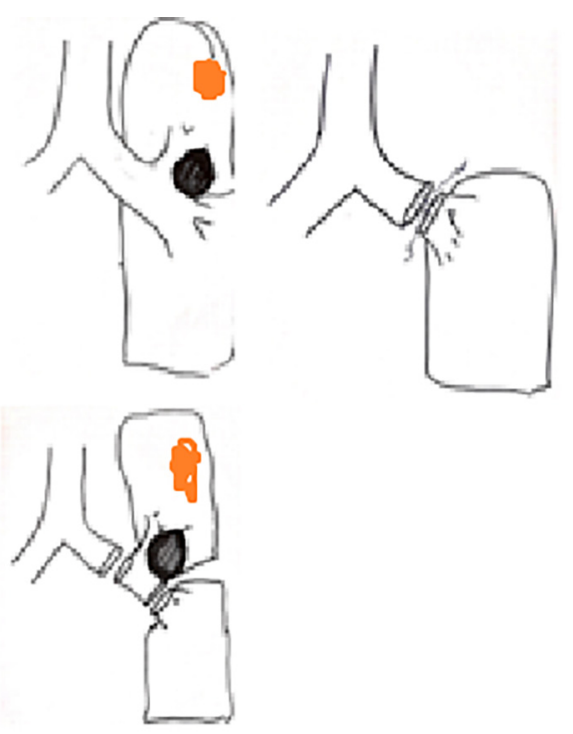

Figure 6 Drawing of the technique.

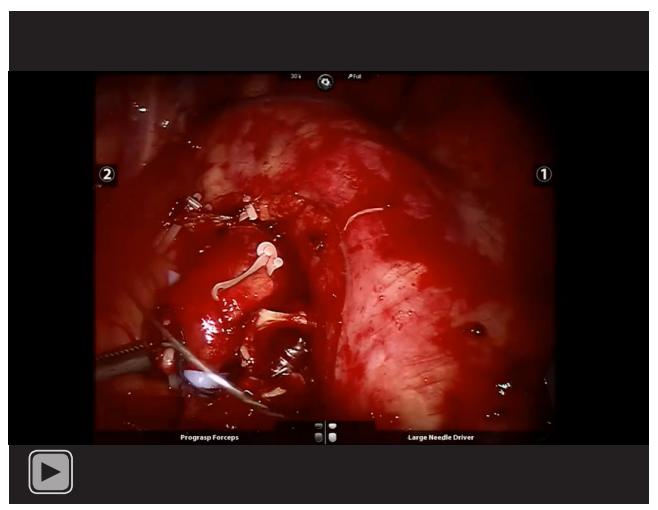

Video 2 Left upper sleeve lobectomy.

where the most anterior port is utilized for assistance and for left arm common use. The planned resection in this case, where the station 11 node of concern is illustrated in black coloring, is pictured in Figure 6.

Pulmonary arteries were divided with hem-O-lock clip applier and distally with laparoscopic vessel sealer. Superior pulmonary vein was divided with a vascular stapler. Then the bronchus was isolated and divided with a scalpel. The surgical bronchial margin was positive. We subsequently performed a sleeve resection of the left main bronchus and origin of left lower lobe bronchus. The anastomosis was performed again with the standard technique described above. Two sutures at the most inferior and posterior part of the anastomosis were placed. A prostate bulldog clamp

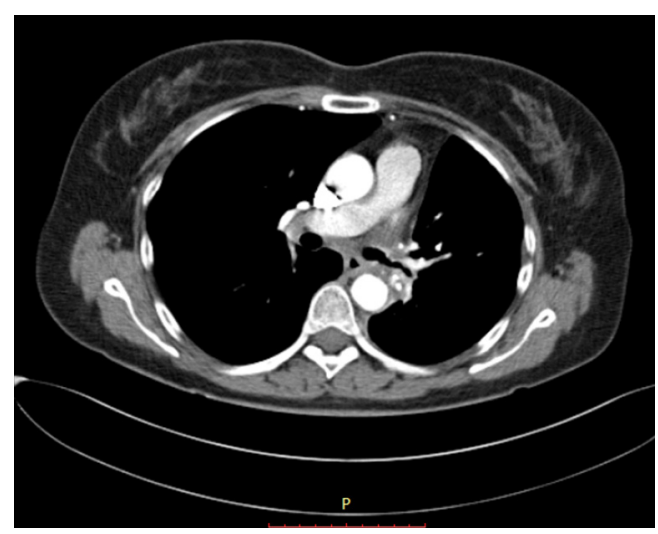

Figure 7 Post-operative images of left upper sleeve lobectomy with $\mathrm{N} 1$ positivity.

was placed on one of the sutures in order to pull the suture. Utilizing the other suture, we performed a continuous running suture technique and anastomosed the medial part of the bronchus. After we finished anteromedial and the portion of the superior aspect, we started with the posterior suture and completed the anastomosis. Both sutures were tied to each other. An air leak test and deflation test was performed (Video 2). We performed a bronchoscopy for clearing of the blood and secretions after the dual lumen intubation tube was exchanged for the \#8 single lumen endotracheal tube. No complications occurred and patient discharged on day 6. Chest CT in the following month demonstrated a nice, patent bronchial lumen (Figure 7).

\section{Discussion}

In the past two decades, video thoracoscopy has become a primary surgical technique for performing almost all thoracic surgical operations. Technological advances in minimally invasive surgery have made the performance of a sleeve resection possible with VATS (13). However, sleeve lobectomy via VATS did not gain as much ground when compared to the rise of robotic surgery in this regard. Robotic surgery, currently via the da Vinci surgical system (da Vinci System Intuitive Surgical, Sunnyvale, CA, USA) is an extension of minimally invasive thoracic surgery. Better flexibility due to the use of EndoWrist instruments, more intuitive movements, and high-definition with threedimensional viewing are some of the superiorities of robotic surgery, which increase the performance of a surgeon to provide negative bronchial margins (14). Robotic sleeve resection was first performed in a human cadaver in 2006 (15). 
The robotic pulmonary sleeve resection is currently becoming a common procedure $(16,17)$. As the indications have expanded, the short-term results demonstrate that robotic sleeve resections are technically feasible, a complete resection is possible, and perioperative outcomes are acceptable (18).

In a recently published report authors share their experiences in 67 patients with centrally located lung cancer. They suggested anastomosis of the cartilaginous and membranous parts completed by a half-continuous Prolene suture with two separate sutures. Our very first experience was same with this group, however we changed to Vi lock 3-0 sutures with increasing experience. In our experience, we demonstrated that the first sleeve lobectomy operation with robotic surgery has been a benchmark. The quality, type, and duration of surgeries have varied following the first sleeve lobectomy operation utilizing the robotic surgical technique (19). Other authors $(18,20,21)$ used the both interrupted or continuous sutures.

The mortality and the morbidity rates in the largest experience were $0 \%$ and $20.6 \%$, respectively. Postoperative complications included atelectasis requiring bronchoscopy, subcutaneous emphysema, arrhythmia, and prolonged air leak (22).

In our early experience between December 2014 and August 2018 out of a total 108 lung cancer patients, 7 patients underwent primary bronchial closure and 9 patients underwent bronchial sleeve resections. We compared these 16 patients with those of 92 lobectomy patients. No statistical differences were found in duration of surgery, intraoperative bleeding, length of postoperative stay, complication rate, and readmission rate. No patients developed a bronchial fistula in both groups (23).

Robot increases surgical technical capacity of the surgeons. However, bronchial sleeve resections and anastomosis are mostly published on single case basis. Rarely they are published as case series. In a period of 2 years, A group from Taiwan operated on 6 patients 5 with squamous cell carcinoma, and 1 with a carcinoid tumor (21). The duration of surgery was longer than conventional literature findings 436.7 [255-745] minutes as well as intensive care unit stay and hospital stay were 3.7 days and 11.3 days, respectively. They noted, one pneumonia and one anastomosis stricture as complications. No mortality and morbidity and conversion to open were recorded (21). Their series supported the idea of complex thoracic surgery could be performed with robotic surgical devices. We have published our early experience with either primary closure or sleeve lobectomies with robotic surgery (23). Our results demonstrated good outcomes. There are a couple of teaching techniques with videos $(24,25)$. Surgeons who want to get into more details about robotic sleeve resections could observe the mentioned videos $(24,25)$. However, sometimes a central lung cancer can invade both the bronchus and the pulmonary artery. A bronchial sleeve and pulmonary arterial angioplasty or arterial sleeve, basically a double-sleeve lobectomy may be needed. Double sleeve lobectomy with minimally invasive surgery is a challenging surgery by conventional thoracotomy. Although VATS has been increasingly reported for double sleeves in the last couple of years, RATS performance has been very rarely reported (26). We believe Robotic surgery may have a real potential in performing double sleeve resections including complex vascular reconstructions.

\section{Acknowledgments}

Funding: None.

\section{Footnote}

Provenance and Peer Review: This article was commissioned by the Guest Editor (Jean-Marc Baste) for the series "Robotic Assisted Thoracic Surgery: Advanced Procedures in Lung and Mediastinum: from Postinduction TTT (immunotherapy) to Sleeve Resection, Complex Segmentectomies and Extended Thymectomy for Myasthenia Gravis" published in fournal of Visualized Surgery. The article has undergone external peer review.

Reporting Checklist: The authors have completed the CARE reporting checklist. Available at https://jovs.amegroups. com/article/view/10.21037/jovs-2020-04/rc

Conflicts of Interest: All authors have completed the ICMJE uniform disclosure form (available at https:// jovs.amegroups.com/article/view/10.21037/jovs-202004/coif). The series "Robotic Assisted Thoracic Surgery: Advanced Procedures in Lung and Mediastinum: from Postinduction TTT (immunotherapy) to Sleeve Resection, Complex Segmentectomies and Extended Thymectomy for Myasthenia Gravis" was commissioned by the editorial office without any funding or sponsorship. AT serves as an unpaid editorial board member of Fournal of Visualized Surgery from June 2015 to May 2021. The authors have no other conflicts of interest to declare. 
Ethical Statement: The authors are accountable for all aspects of the work in ensuring that questions related to the accuracy or integrity of any part of the work are appropriately investigated and resolved. All procedures performed in this study were in accordance with the ethical standards of the institutional and/or national research committee(s) and with the Helsinki Declaration (as revised in 2013). Written informed consent were obtained from the patients for publication of this case report and accompanying images. A copy of the written consent is available for review by the editorial office of this journal.

Open Access Statement: This is an Open Access article distributed in accordance with the Creative Commons Attribution-NonCommercial-NoDerivs 4.0 International License (CC BY-NC-ND 4.0), which permits the noncommercial replication and distribution of the article with the strict proviso that no changes or edits are made and the original work is properly cited (including links to both the formal publication through the relevant DOI and the license). See: https://creativecommons.org/licenses/by-nc-nd/4.0/.

\section{References}

1. Owen RM, Force SD, Gal AA, et al. Routine intraoperative frozen section analysis of bronchial margins is of limited utility in lung cancer resection. Ann Thorac Surg 2013;95:1859-65.

2. 2. Tomaszek SC, Kim Y, Cassivi SD, et al. Bronchial resection margin length and clinical outcome in non-small cell lung cancer. Eur J Cardiothorac Surg 2011;40:1151-6.

3. Rami-Porta R, Wittekind C, Goldstraw P, et al. Complete resection in lung cancer surgery: proposed definition. Lung Cancer 2005;49:25-33.

4. Wind J, Smit EJ, Senan S, et al. Residual disease at the bronchial stump after curative resection for lung cancer. Eur J Cardiothorac Surg 2007;32:29-34.

5. Ma Q, Liu D. VATS right upper lobe bronchial sleeve resection. J Thorac Dis 2016;8:2269-71.

6. Branscheid D, Beshay M. The technique of sleeve resection on the bronchial and pulmonary vascular tree. Chirurg 2013;84:459-68.

7. Nowak K, Karenovics W, Nicholson AG, et al. Pure bronchoplastic resections of the bronchus without pulmonary resection for endobronchial carcinoid tumours. Interact Cardiovasc Thorac Surg 2013;17:291-4; discussion 294-5.

8. Radosavljevic V, Gardijan V, Brajkovic M, et al. Lung hamartoma--diagnosis and treatment. Med Arch 2012;66:281-2.

9. De leyn P, Decaluwe H. Sleeve lobectomy. In Shield's General Thoracic Surgery 8th edition. In: Locicero L, Feins RH, Colson Y, et al. editors. Philadelphia: Wolters Kluwer, 2019.

10. Cosgun T, Kaba E, Ayalp K, et al. Bronchial sleeve anastomosis and primary closures with the da Vinci system: an advanced minimally invasive technique. Video-assist Thorac Surg 2017;2:49-6.

11. Bilgic E, Watanabe Y, Nepomnayshy D, et al. Multicenter proficiency benchmarks for advanced laparoscopic suturing tasks. Am J Surg 2017;213:217-21.

12. Lin MW, Kuo SW, Yang SM, et al. Robotic-assisted thoracoscopic sleeve lobectomy for locally advanced lung cancer. J Thorac Dis 2016;8:1747-52.

13. Mahtabifard A, Fuller CB, McKenna RJ. Video assisted thoracic surgery sleeve lobectomy. Case series. Ann Thorac Surg 2008;85:S729-32.

14. Paul S, Altorki NK, Sheng S, et al. Thoracoscopic lobectomy is associated with lower morbidity than open lobectomy: a propensity matched analysis from the STS database. J Thorac Cardiovasc Surg 2010;139:366-78.

15. Ishikawa N, Sun YS, Nifong LW, et al. Thoracoscopic robot-assisted bronchoplasty. Surg Endosc 2006;20:1782-3.

16. Schmid T, Augustin F, Kainz G, et al. Hybrid video assisted thoracic surgery-robotic minimally invasive right upper lobe sleevelobectomy. Ann Thorac Surg 2011;91:1961-5.

17. Zhao Y, Jiao W, Ren X, et al. Left lower lobe sleeve lobectomy for lung cancer using the Da Vinci surgical system. J Cardiothorac Surg 2016;11:59.

18. Pan X, Gu C, Wang R, et al. Initial Experience of robotic Sleeve Resection for Lung Cancer Patients. Ann Thorac Surg 2016;102:1892-7.

19. Cosgun T, Kaba E, Ayalp K, et al. Successful Sleeve Resection as a Marker for Proficiency for Robotic Pulmonary Resection. Thorac Cardiovasc Surg 2019. [Epub ahead of print].

20. Cerfolio RJ. Robotic sleeve lobectomy: technical details and early results. J Thorac Dis 2016;8:S223-6.

21. Lin MW, Kuo SW, Yang SM, et al. Robotic-assisted thoracoscopic sleeve lobectomy for locally advanced lung cancer. J Thorac Dis 2016;8:1747-52.

22. Jiao W, Zhao Y, Qiu T, et al. Robotic Bronchial Sleeve Lobectomy for Central Lung Tumors: Technique and Outcome. Ann Thorac Surg 2019;108:211-8.

23. Coşgun T, Kaba E, Ayalp K, et al. An antiquated 
contraindication for minimally invasive lung surgery: No place to staple the bronchus. Turk Gogus Kalp Damar Cerrahisi Derg 2019;27:521-5.

24. Aprile V, Zirafa CC, Ilenia C, et al. Robotic Right Upper Lobe Sleeve Lobectomy. April 2018. doi: 10.25373/ ctsnet.6137384. Available online: https://www.ctsnet.org/

doi: 10.21037/jovs-2020-04

Cite this article as: Toker A, Kaba E, Herron R, Ayalp K. Case report: left upper and left lower sleeve lobectomies with robotic surgery. J Vis Surg 2022;8:17. article/robotic-right-upper-lobe-sleeve-lobectomy

25. Durand M. Four-arm robotic sleeve right upper lobectomy. Ann Cardiothorac Surg 2019;8:286-7.

26. Qiu T, Zhao Y, Xuan Y, et al. Robotic-assisted doublesleeve lobectomy. J Thorac Dis 2017;9:E21-5. 